\title{
The escape of trapped electrons in the decay phase of solar flare
}

\author{
Jing Huang \\ Key Laboratory of Solar Activity, National Astronomical Observatories, Chinese Academy of \\ Sciences, Beijing, 100012, China \\ email: huangj@bao.ac.cn
}

\begin{abstract}
From the observations of radio and HXR bursts, the escape rate of energetic electrons trapped in the flare loops is studied based on the trap-plus-precipitation model for the kinematics of energetic electrons in solar flares. Coulomb collision is regarded as the main pitch angle scattering of trapped electrons in the decay phase of the event on 2004 December 1 . The escape rate of trapped electrons decreases firstly and then increases, which indicates the evolution of the plasma density in the flare loops during the decay phase.
\end{abstract}

Keywords. solar flare, energetic electrons, escape

\section{Introduction}

Energetic electrons produce plenty of bursts when they transport in the solar atmosphere. Among these burst events, radio and HXR bursts are normally observed by spatial and ground-based telescopes. Radio burst from Gyrosynchrotron emission and hard Xray burst from thin-target Bremsstrahlung emission may be used to infer the electron distribution and its evolution in time (Bastian et al. 1998). The trap-plus-precipitation model is generally adopted to describe the transport of energetic electrons (Aschwanden 1998). Because of the magnetic mirrors of flare loops, electrons are trapped and accumulated in the flare loops. In the decay phase, the energetic electron injection decreases obviously. The trapped electrons and the ones escaping from trap are the main emitters of radio and HXR emission in the decay phase. The escape of trapped electrons plays an important role in the evolution of electrons producing radio and HXR bursts. The ratio of the escape rate caused by energy loss to pitch angle scattering is about 0.3 (Hundson 1972) or 0.5 (Trubnikov 1965). The mechanism of pitch angle scattering includes coulomb collision, resonant scattering and diffusive scattering.

\section{Data reduction}

The radio burst at $7.6 \mathrm{GHz}$ is provided by the Solar Broad-band Radio Spectrometer (SBRS) of China (Fu et al. 2004) and the HXR emission at 25-50 keV is from the Reuven Ramaty High-Energy Solar Spectroscopic Imager (RHESSI; Lin et al. 2002). There are several parameters related to radio and HXR emission. It is hard to deduce the exact amount of number density of energetic electrons. Hence, the radio and HXR light curves could be used as the template of number density of energetic electrons producing radio and HXR bursts. It is assumed that the number density of radio-producing electrons is 20 times of that for HXR emission. By ignoring the injection in the decay phase and assuming the first precipitating rate (q) being invariable, the escape rate of trapped 


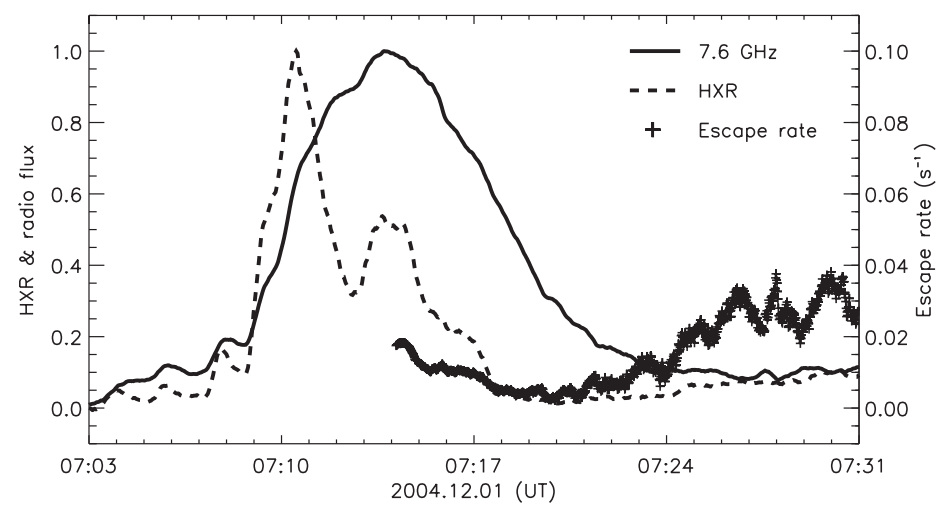

Figure 1. The radio flux at 7.6 GHz (solid line), HXR flux at 30-70 keV (dashed line) and the escape rate of trapped electrons (+) of event on 2004 Dec. 1.

electrons deduced from the trap-plus-precipitation model is:

$$
\nu(t)=\log \frac{(q+1) \cdot n_{r}(t-1)}{q \cdot n_{r}(t)+n_{r}(t-1)-n_{h}(t)}
$$

where $n_{r}$ and $n_{h}$ are the number density of energetic electrons producing radio and HXR emission, respectively.

\section{Results and conclusions}

The radio burst at $7.5 \mathrm{GHz}$ peaks at $07: 13: 44$ with a 192 -s delay from the HXR maximum and has a more gradual decay as shown in Figure 1. This is due to the accumulation of trapped electrons in the flare loops during the whole process. Huang \& Yan (2009) have fitted the radio emission at six frequencies using HXR flux as the injection function and a increasing escape rate. Coulomb collision is proposed as the pitch angle scattering mechanism for the trapped electrons and $\nu_{p} \approx 10 n_{9} E^{-3 / 2} s^{-1}$, where $\mathrm{E}$ is in units of keV and $n_{9}$ is the ambient plasma density in $10^{9} \mathrm{~cm}^{-3}$. In this work, the escape rate deduced from radio and HXR observation decreases firstly and then increases during the decay phase. It suggests that the plasma density around the resonant layer decreases firstly and then increases. The first decrease may be due to the continues expanding of post flare loops and the later increase could be the signature of chromosphere evaporation. The latter is more important on the change of local plasma.

\section{Acknowledgements}

This work was supported by NSFC grants 11103039, 10921303, 10903013, 11273030, the National Basic Research Program of MOST (grant 2011CB811401).

\section{References}

Aschwanden, M. J. 1998, ApJ, 502, 455

Bastain, T. S., Benz, A. O., \& Gary, D. E. 1998, ARA $\& A$, 36, 131

Fu Q. J. et al. 2004, Sol. Phys., 222, 167

Huang, J. \& Yihua Yan 2009, ApJ, 705, 1063

Hudson, H. S. 1972, Sol. Phys., 24, 414

Lin, R. P. et al. 2002, Sol. Phys., 210, 3

Trubnikov, B. A. 1965, Rev. Plasma Phys., 1, 105 